\title{
A Review of Metacognition in the diagnosis and treatment of Obsessive Compulsive Disorder
}

\author{
Kaneenica Ninawe ${ }^{1}$, Hemal Shroff ${ }^{2}$ \\ ${ }^{1,2}$ Center for Health and Social Sciences, School of Health System Studies, Tata Institute of Social \\ Sciences, Mumbai. \\ E-mail - kaneenica@gmail.com \\ Corresponding Author - Kaneenica Ninawe
}

\begin{abstract}
Cognitive theories are more prominent among the theories that explain the etiology and maintenance of OCD as well as among those that offer treatment options. Metacognition is a concept included within the newer theories and models within the cognitive perspective. This is a review of literature with particular focus on the concept of metacognition in cognitive models of OCD, metacognitive measurement tools and metacognitive treatment options. Although there have been metacognitive elements within the cognitive theories for a long time, it is only recently that they have been given importance and considered as separate variables. Research suggests that metacognition plays a central role in the etiology and maintenance of OCD. There are multiple valid and reliable metacognitive tools that are available for diagnostic and treatment purposes. Metacognitive treatments offer promising results for patients with OCD. However, there is a need for more robust empirical evidence using large-scale and longitudinal studies to establish its role more concretely.
\end{abstract}

Key Words: Metacognitive Therapy, Inference Based Therapy, Acceptance and Commitment Therapy, Mindfulness Based Cognitive Therapy, Metacognitive Questionnaire-30.

(Paper received $-9^{\text {th }}$ May 2017, Peer review completed $-1^{\text {st }}$ August 2017, Accepted $-8^{\text {th }}$ August 2017)

\section{INTRODUCTION}

Obsessive Compulsive Disorder (OCD) is characterized by the presence of obsessions, which are recurrent and persistent thoughts, urges, or images that are experienced as intrusive and unwanted. OCD is also characterized by compulsions, which are repetitive behaviors or mental acts that an individual feels driven to perform in response to an obsession or according to rules that must be applied rigidly [1]. OCD is a reasonably common disorder, with a 12 -month prevalence of $1.2 \%$ and a lifetime prevalence of $2.3 \%$ [2]. Cognitive models are amongst the most prominent ones in explaining the etiology and maintenance of OCD.

Metacognitive theory is a newer approach within the cognitive models. The focus of this review is restricted to the role of metacognition in theories of $\mathrm{OCD}$, instruments for measurement of metacognitive factors in relation to OCD and metacognitive treatment for those with a diagnosis of OCD along with supporting research evidence for each one of them. Metacognition as a concept was initially elaborated by Flavell [3], and referred to beliefs about thinking and strategies used to control and regulate thinking. Metacognition therefore is in simple words, thinking about thinking. Metacognition is a general term that can be broken down into three main areas: metacognitive knowledge, experiences, and strategies [3-4]. The first area refers to information stored in memory in a declarative form such as false declarative beliefs, or in other words, negative metacognitive beliefs. In OCD, this might be the belief that washing rituals 
protect one's family from harm. The second area (metacognitive experiences) refers to the experience of an individual with OCD who might report the feeling of just knowing when to refrain from or complete a ritual. The final area refers to strategies used to regulate and control cognitions, for example an individual with OCD suppressing an unwanted thought [5].

\section{METACOGNITION IN THEORETICAL MODELS OF OCD}

Cognitive Models have also incorporated metacognitive factors right from the beginning though they were understood as being cognitive in nature. Salkowkis [6] posited that not only intrusive thoughts, but the appraisal of these intrusions is the core issue in OCD. These thoughts are subject to misinterpretation; as a signal that there is serious risk of harm to the self or others and the responsibility for such harm, as well as its prevention rests with them. He conceptualized responsibility on two levels: responsibility beliefs and responsibility appraisals. This appraisal itself is a metacognitive concept. Rachman [7-9] on the other hand put catastrophic misinterpretation of the significance of thoughts at the center of his theory. According to him, neutral stimuli are converted into threatening stimuli if the content of the intrusive thought has particular meaning to the person. The magnification of the significance of one's intrusive thoughts is an important construct in this theory as it is presumed that the individual will assume that their intrusive thoughts reveal something important about their moral character. Rachman also highlighted a cognitive process whereby one's thoughts and actions become fused together. Rachman [8-10] termed this phenomenon Thought-Action Fusion (TAF). He further proposed that TAF in combination with inflated responsibility beliefs, served to increase the catastrophic misinterpretation of the significance of the unwanted thoughts. TAF is defined as the cognitive process whereby individuals with OCD experience thoughts and actions concerning harm as equivalent and see themselves as equally responsible for thinking as for acting [7]. TAF is suggested to have two components; Moral-TAF (TAF-M) and Likelihood-TAF (TAF-L) [11-12]. TAF-M refers to the belief that having an unacceptable thought is almost the moral equivalent of carrying out that particular behavior [13]. TAF-L refers to the belief that the occurrence of a thought will increase the likelihood that the specific negative event will occur [14]. Both these concepts are also metacognitive in nature. In line with the above-mentioned models, the Obsessive- Compulsive Working Group [15-17] defined six faulty belief domains that are important contributors in OCD. The belief domains that were hypothesized to be influential were 1) an inflated sense of responsibility, 2) overestimation of threat, 3) over- importance of thoughts, 4) emphasis of thought- control, 5) intolerance of uncertainty and 6) perfectionism. The belief domain referred to as over-importance of thoughts refers to the process of being unable to dismiss naturally occurring intrusive thoughts and instead ascribing importance and meaning to them, which in itself, is a metacognitive process [5].

Clark and Purdon [18] revised the earlier cognitive frameworks and focused upon the role of metacognitive processes in their emphasis on the need for control over thoughts as an important domain in OCD. They stressed that individuals with OCD misinterpret the consequences of failure in controlling unwanted intrusive. They explained that when individuals with OCD are unable to suppress the unwanted thought, they become even more focused on the occurrence of the thoughts and engage in greater efforts to suppress them, maintaining the obsessive cycle. Though these appraisals can be viewed as Metacognitive knowledge, the authors viewed it as a cognitive process.

Wells and Matthews [19] and Wells [20] put forth the metacognitive model of emotional disorders focusing especially on OCD. The metacognitive model of OCD, introduced by Wells and Mathews [1921] is also known as Self-Regulatory Executive Function (S- REF) Model. According to the S- REF model, psychological disorders may develop and be maintained because of the functioning of a cognitive processing system called the 'Cognitive Attentional Syndrome' (CAS) operating at multiple levels. The CAS consists of three components that are overlapping; a perseverative thinking style characterized by worry and rumination, unhelpful hypervigilant attention to threat, and counterproductive coping which prevents regulation of cognition. Positive and negative beliefs about thinking guide the appraisals. Thus the model focuses more on the process rather than the content of thinking. Beliefs in S- REF are 
conceptualized into two broad areas:- 1) beliefs surrounding the importance and power of the thoughts and 2) beliefs about the need to control these thoughts and engage in ritualistic behaviours [22]. Wells [4] argues that three types of metacognitive knowledge are important in the etiology and maintenance of symptoms: thought fusion beliefs, beliefs about the need to perform rituals, and criteria that signal rituals can be stopped. Individuals with OCD have a tendency to focus more attention on their own thoughts, in turn, making them more unique and significant and thus, facilitating the ease of detection of these intrusive thoughts. Their metacognitive beliefs regarding the importance and meaning of the thoughts guide the negative appraisal of these thoughts. The negative interpretation of the intrusive thought as being potentially harmful, prompt certain behavioral responses in order to deal with the perceived danger. This behavioral response could be in the form of neutralization or compulsive rituals [4].

A number of studies have been conducted to understand the role of metacognition in the etiology and maintenance of OCD. Studies suggest that thought-action fusion beliefs and beliefs about the uncontrollability and danger of thoughts correlate significantly with OCD symptoms. Emmelkamp and Aardema [23] found that meta-cognitive beliefs (including TAF) were related to scores on obsessivecompulsive measures in a community sample. Brown \& Naragon-Gainy [24] reported that TAF, a metacognitive variable was found to have a significant path to OCD in regression analysis. Also, Wells and Papageorgiou [25] demonstrated that meta-cognitive beliefs predicted obsessions, independent of general proneness to worry. Janeck, Calamari, Riemann and Heffelfinger [26] examined the metacognitive domain of Cognitive Self-Consciousness (CSC i.e., the tendency to be aware of and monitor thinking), and reported that CSC was significantly correlated with obsessive-compulsive symptoms, with higher scores in OCD patients than anxious clinical controls and a non-clinical control group in an adult population in the U.S. They explained that CSC, which is a person's tendency to excessively reflect upon one's own cognitive processes increases the likelihood of appraising an intrusive thought more negatively, fostering the over-importance of thoughts leading to the likelihood of developing and maintaining OCD. In a study by Sica, Steketee, Ghisi, Chiri, \& Franceschini [27] negative beliefs about worry, which focused on uncontrollability and danger were consistently associated with worry and obsessive symptoms, after controlling for the initial level of both worry and obsessionality. This was true even after a four month interval. In addition, they found that positive beliefs about worry predicted maladaptive coping styles, whereas cognitive self-consciousness and thought strategies aimed at distraction predicted adaptive coping styles. In another study with an Indian sample, the researchers found cross-sectional and causal relationships between obsessive compulsive beliefs, metacognitive beliefs and OCD [28]. Furthermore, change in metacognitive scores has been found to be better predictor of exposure and response prevention treatment outcome than changes in responsibility or perfectionism beliefs. This supports the metacognitive model over other dominant theories [29]. Myers, Fisher, and Wells [30] tested the relative contribution of each of the three metacognitive domains proposed by Wells [4] (viz. fusion beliefs, beliefs about rituals and stop signals) in predicting obsessive-compulsive symptoms in a sample of 238 university students. Using hierarchical regression analysis, they controlled for worry as well as threat, responsibility, and perfectionism. They found that the three metacognitive belief domains explained unique variance in obsessive compulsive symptoms. The study was limited by the use of a non-clinical sample and the use of some unpublished measures. This study was later replicated and extended by Solem, Myers, Fisher, Vogel, and Wells [31], who found a similar pattern of results among a sample of 304 non-clinical participants. All three metacognitive domains were significantly correlated with obsessive-compulsive symptoms, but only fusion beliefs and beliefs about rituals predicted obsessive-compulsive symptoms after controlling for worry, threat, and non metacognitive beliefs. Once again, conclusions were limited by the cross-sectional nature of the study and the use of a non-clinical sample. However, the authors included a relatively small OCD clinical sample in the first part of the study where they compared scores on metacognitive measures and found significant differences between the OCD and the non-clinical sample. Furthermore, they found that fusion beliefs and beliefs about rituals were significant predictors of obsessive-compulsive symptoms in the clinical sample. 


\section{METACOGNITION ASSESSMENT IN OCD}

There are a number of standardized self-report tools that measure different aspects of metacognition in relation to OCD. Some have been validated with the clinical sample while some have not. Some of them were originally developed as measures of cognitive factors (which include metacognitive factors) and some were developed to measure metacognitive factors themselves.

\section{Obsessive Beliefs Questionnaire (OBQ/OBQ-44):}

There are two versions of this scale, OBQ and a shorter version (OBQ-44) that assess belief domains that have been proposed to be important in the etiology of OCD. The original OBQ has 87 items, all rated on a 7 point scale from 1 (strongly disagree) to 7 (strongly agree). It measures 6 subscales: (a) inflated responsibility:- the belief that one has power to bring about or prevent subjectively crucial negative outcomes; (b) the over importance of thoughts:- the belief that the mere presence of a thought indicates that it is important; (c) the importance of controlling one's thoughts:- the overvaluation of the importance of exerting complete control over one's thoughts, and the belief that it is both possible and desirable; (d) overestimations of threat:- an exaggeration of the probability and severity of harm; (e) intolerance of uncertainty:- beliefs that it is a necessity to be certain, that one has a poor capacity to cope with unpredictability, and that one finds it difficult to cope in ambiguous situations; and (f) perfectionism:- the tendency to believe there is a perfect solution to every problem, that performing an act without mistakes is both possible and necessary, and the belief that even minor mistakes will have serious consequences [15]. However, the six scales were highly inter-correlated, with correlation values ranging from 0.59 to 0.81 in a combined sample of students, community participants, and individuals with OCD. Hence an exploratory factor analysis was conducted to create a shorter and more reliable tool.

The OBQ-44 has 44 items, all rated on a 7 point scale from 1 (strongly disagree) to 7 (strongly agree). The OBQ-44 has been found to show good internal consistency and criterion-related validity in both clinical and non-clinical samples [32-33]. The three subscales in this measure are: (a) responsibility/threat, (b) perfectionism/certainty, and (c) importance/control of thoughts. The importance/control of thoughts subscale has been construed as measuring a metacognitive construct. This subscale significantly predicts obsessing and neutralizing symptoms in OCD and also distinguishes individuals with OCD from those having other anxiety disorders [33-34].

\section{Metacognition Questionnaire (MCQ/MCQ-30):}

There are two versions of this questionnaire. MCQ [35] was originally a 65 -item scale developed to assess several dimensions of metacognition, thought to be relevant to OCD following the conceptual analysis offered by the S-REF theory. MCQ-30, a brief version of MCQ, is a 30-item self-report scale measuring beliefs about thinking. Responses are sought on a four-point Likert scale ranging from 1 (do not agree) to 4 (agree very much). Five subscales exist: (a) positive beliefs about worry; (b) negative beliefs about the controllability of thoughts and corresponding danger; (c) cognitive confidence; (d) negative beliefs about thoughts in general/need to control thoughts; and (e) cognitive self-consciousness. Good psychometric properties have been reported [36-37]. It has been translated into several languages, including Spanish [38], Turkish [39], Italian [40] and Korean [41] with acceptable psychometric properties.

\section{Interpretation of Intrusions Inventory (III):}

The III [16] is a 31- item scale which assesses immediate appraisals of intrusive thoughts. The III is designed to have three subscales: appraisals of responsibility, over importance of thought intrusions, and control of intrusions. Respondents are first provided with definitions and examples of unwanted egodystonic mental intrusions and are then asked to record two recent intrusive thoughts, impulses, or images that they have experienced. They are also asked to record the recency, frequency, and distress associated with these intrusions. Respondents are then required to rate 31 statements (e.g., "I would be a better person if I didn't have this thought") using a scale from 0 ("I did not believe this idea at all") to 100 ("I was completely convinced this idea was true"). For data analysis the 100-point scale is bought down to a 
10-point scale, so that, total scores range from 0 to 310 . Studies have demonstrated that the III has excellent reliability in clinical samples, non-anxious clinical samples, as well as student and community samples and also has been observed to have moderate test-retest reliability in non-clinical and clinical populations $[17,42]$. A review of the literature suggests that this scale is used more in research rather than in clinical practice.

\section{Meta-Cognitive Beliefs Questionnaire (MCBQ):}

The MCBQ [43] is a 67-item self-report questionnaire that assesses beliefs about the importance of control and negative consequences associated with unwanted, ego-dystonic intrusive thoughts, images and impulses. MCBQ consists of three dimensions; (a) perception of the possibility and importance of mental control, (b) perceived negative consequences resulting from a failure to control intrusive thoughts, and (c) perceived positive attributes of unwanted mental intrusions [43]. Respondents are provided with definitions and examples of unwanted mental intrusions and are then asked to rate how strongly they agree or disagree with a range of belief statements. Each of the belief statements is asked to be written down so that reference is made to the unwanted intrusive thoughts. Respondents are asked to rate their level of agreement on a 7 point bipolar scale ranging from 'totally disagree' $(-3)$ to 'neutral' (0) to 'totally agree' (+3). The MBCQ has been reported to have good concurrent and discriminant validity. MCBQ scale scores were found to significantly predict obsessional, but not anxious or depressive, symptomatology [43].

\section{The Thought Fusion Instrument (TFI):}

The TFI [44] is a 14-item self-report measure designed to assess three types of thought fusion beliefs: (a) Thought-Event Fusion (e.g., "My thoughts alone have the power to change the course of events"); (b) Thought-Action Fusion (e.g."If I have thoughts about harming myself, I will end up doing it"); and (c) Thought-Object Fusion (e.g., "My feelings can be transferred into objects"). Each item can be rated from 0 to 100 . TFI has good internal consistency with Cronbach's alphas ranging from 0.72 to 0.80 for the three subscales and 0.89 for the full scale. Moderate test-retest reliability, and support for its convergent and discriminant validity was also reported [44]. The TFI has been used in several studies to assess metacognition in non-clinical as well as clinical samples [30, 31, 45].

\section{Thought-Action Fusion Scale (TAF):}

The TAF [12] is a 19-item self-report measure to assess thought-action fusion beliefs. Respondents have to answer on a 5-point scale ranging from 0 (disagree strongly) to 4 (agree strongly). TAF-total scores thus range from 0 to 76 . The scale is comprised of two components; (a) thought-action fusion moral which refers to the belief that having a sinful thought is the same as indulging in a sinful act and (b) likelihood beliefs which refers to the belief that thinking about an undesirable or distressing event, increases the probability of that event. TAF has been studied extensively in relation to OCD. Studies have shown that the TAF total, and moral and likelihood subscales have good internal consistency and correlate with selfreports of obsession symptoms [46, 12]. It has been translated into several languages including Dutch [46], Turkish [47] and Icelandic [48].

\section{METACOGNITIVE TREATMENT APPROACHES FOR OCD}

A number of treatment approaches are available for OCD within the Metacognitive framework.

\section{Metacognitive Therapy (MCT):}

Metacognitive therapy, in line with Wells' Metacognitive theory focuses on the maladaptive thinking processes as against the specific maladaptive content of the thoughts. The main aim of MCT is to enable patients to move from treating their thoughts about their obsessions and compulsions as facts, to being able to objectively evaluate their obsessions as merely mental events not requiring further processing. MCT thus attempts to modify metacognitive beliefs about obsessions and rituals, reduce levels of rumination, worry, self-focused attention and replace maladaptive coping strategies with adaptive coping strategies 
which facilitate the development of functional processing [45]. Instead of exposure exercises used in Cognitive Behaviour Therapy (CBT), in MCT patients are made aware of their higher-level metacognitive functioning errors and then behavioral experiments are engaged in to assist them in interrupting maladaptive coping strategies such as thought suppression [49]. Detached Mindfulness (DM) is taught to the patient as means of becoming aware and modifying metacognitive beliefs about obsessions and rituals. $\mathrm{DM}$ is used to establish a more appropriate mode of processing, improving executive control, removing cognitive perseveration in the form of worry/rumination [5]. DM is not the same as mindfulness meditation. Wells $[4,50]$ explained that while there is some overlap between the two, DM differs from mindfulness meditation in a number of ways. DM involves specific awareness of cognition rather than the broader context of attention to present moment experiences. DM includes detachment aimed at helping the patient to achieve a metalevel of experience in which thoughts and internal events are not fused with the sense of self and external reality. Detached mindfulness is achieved by giving the patient practice of the strategy with emotionally neutral material and is then practiced in response to their preoccupation within the session [45].

A number of studies have been conducted to explore the efficacy of MCT. In a study by Fisher and Wells [45] case series approach was used wherein 4 OCD patients with diverse presentations of OCD were given MCT over 12 individual sessions of not more than 1 hour each. A substantial reduction was found on all outcome measures, after treatment as well as at 3-month and 6-month follow-ups. Similar results were found in an open trial for group MCT for 8 adults with OCD [51] and in another case series study on patients reporting from pure obsessions (obsessions without overt compulsions) in Iran [52]. In another study MCT was applied in the treatment of pediatric OCD cases [53]. Ten children and adolescents were randomly assigned either to an Exposure Response Prevention (ERP) group or a MCT group. A significant reduction in symptoms were seen after treatment and at 3-month and 2-year follow ups, suggesting that MCT is a promising alternative to ERP with pediatric population also. In one study 19 patients with OCD were allocated randomly to MCT, fluvoxamine or combined MCT-fluvoxamine groups and then compared [54]. Both, the MCT and the combined groups showed significant reductions in symptoms after 10 weeks of treatment as compared to the fluvoxamine group. There was no statistical difference between the MCT and combined group indicating that the addition of medication did not increase the efficiency of MCT. Moritz, Jelinek, Hauschildt and Naber [55] developed and tested a selfhelp treatment called 'My Metacognitive Training for OCD' (myMCT). Recruitment took place over the internet and 86 individuals with OCD were randomly assigned to either a self-help or a wait-list condition. Those in the experimental group were emailed an electronic version of the self-help book (myMCT). Significantly greater reductions were seen in obsessive-compulsive symptoms in participants who received the intervention as compared with those in the wait-list group. This treatment however also contained traditional cognitive restructuring elements and hence must be viewed with caution as a pure test of metacognitive treatment for OCD.

\section{Inference Based Therapy (IBT)}

IBT is based on the Inference Based Approach [56]. IBA postulates that the obsessional chain always begins with a doubting inference achieved through a reasoning process referred to as inferential confusion [57]. Authors postulated that inferential confusion has two components: (a) an investment in remote and often imaginary possibilities in preference to reality and (b) a distrust of the senses and common sense. These two components together, confuse the person with OCD and make him/ her have doubts without any legitimate reasons for such doubts. IBT aims to refute the reasoning causing the primary inference and further attempts to help the person trust their senses and common sense. IBA endorses the idea that "promoting meta-cognitive insight into the distinctive nature and origins of the obsessional narrative helps the person to realign their thinking in the obsessional situation with their thinking before the OCD started and with their thinking in other non-OCD related areas of their lives, and recalibrate their actions accordingly" [56,p.no.420]. Treatment involves a series of steps: (1) illustrating how the doubt goes against perception in the here and now and detracts from common sense; (2) examining the power of the imagination; and (3) utilizing techniques to return the person to the senses and common sense (such as 
constructing an alternative realistic narrative, grounding the person in reality sensing). Metacognitive insight is promoted as the patient is assisted to become aware of their maladaptive general response to intrusive thoughts as opposed to engaging with the content of those thoughts.

In one study, the efficacy of IBT was evaluated for OCD by comparing it with a treatment based on the cognitive appraisal model (CAM) or ERP [58]. The participants ( $N=54)$, were randomly assigned to one of three groups. After 20 weeks of treatment, 44 had completed and all groups showed a significant reduction in outcome measures. Participants with high levels of obsessional conviction showed greater benefit from IBA than from CAM. Aardema and O'Connor [59] evaluated the impact of IBT in an open trial with a sample of 35 patients diagnosed with OCD. Results of the study confirmed that IBT improved the participants' ability to resolve obsessional doubt. Significantly better treatment outcomes were seen in those who had improved their abilities to resolve doubt. A randomized controlled trial has also been done, in which either 24 CBT sessions or 24 IBA sessions were administered to 90 patients with OCD with poor insight [60]. The primary outcome measure was the Yale-Brown Obsessive Compulsive Scale (YBOCS). Secondary outcome measures were level of insight, anxiety and depressive symptoms, and quality of life. The researchers established that patients with OCD with poor insight improve significantly after psychological treatment. The results of this study suggest that patients with OCD with poor insight were effectively treated by both CBT and IBA. IBA was found to be more promising than CBT for patients with more extremely poor insight.

\section{Acceptance and Commitment Therapy (ACT)}

ACT is anchored in the philosophy of functional contextualism and the Relational Frame Theory [61]. The theory basically postulates that individuals learn relationships between stimuli and responses through a number of different processes. It further suggests that these relationships are not necessarily subject to an actual experience with those stimuli. For example, a person with OCD may fear contamination from any door handle because they are similar to door handles on the toilet which are considered to be dirty. ACT comprises six main therapy processes. These processes are not linear, but are tended to within the therapy as and when the need or opportunity emerges. These processes are (1) acceptance; (2) defusion; (3) self as context; (4) contact with the present moment; (5) values; and (6) committed action [62]. In ACT, the person with OCD is helped to see how past attempts to control obsessions have failed and to focus on accepting rather than struggling to stop the obsessional thoughts. Rather than reducing or eliminating symptoms, the therapist asks the client to focus on improvement in quality of life as the goal of therapy. Another significant part of the therapy is to help the clients achieve cognitive defusion, i.e., to see thoughts less literally. Clients are helped to defuse from obsessions by treating them just as thoughts in one's head having little power and meaning or as relatively unimportant words [63]. A range of cognitive exercises are used to help the client to achieve defusion. These exercises are metacognitive in nature. The client is encouraged to thank the mind for the thought and not get involved in it any further, treat the obsession as a number of different things, and engage in mindfulness exercises. Another important concept in ACT is self as context. It refers to the process of helping the client to differentiate his or her inner experiences from self, viewing self as an observer or context in which experiences occur [63]. For instance, a person with OCD would be encouraged to understand that obsessional thoughts are separate from who they are as a person. The defusion part of ACT thus appears to target thought suppression which is a counterproductive metacognitive strategy. Several other treatment aspects are also seen in ACT; however, these are not focused on metacognitive change.

A study by Twohig, Hayes, and Masuda [64] evaluated the effectiveness of an 8-session ACT intervention in a non concurrent multiple-baseline study. Significant reductions in compulsions were noted by the end of treatment for all participants, with results maintained at 3-month follow-up. Positive changes in anxiety and depression were also found for all participants along with expected process changes in the form of decreased experiential avoidance, believability of obsessions, and need to respond to obsessions. In a study the effectiveness of eight sessions of ACT were compared with Progressive Relaxation Training (PRT) in a sample of 79 adult patients with OCD [65]. ACT produced clinically significant changes in OCD severity 
at post treatment and follow-up over PRT. Greater change in depression among those reporting at least mild depression before treatment was also reported. Quality of life improved in both conditions but was marginally in favour of ACT at post treatment. Finally, treatment refusal and drop-out was low in both conditions. Although this study provided evidence for the efficacy of ACT, more large scale trials, longer follow up studies and comparative studies using other treatments for OCD like ERP, CBT and MCT, need to be conducted. The suitability of ACT for scrupulosity OCD was explored by Dehlin, Morrison, \& Twohig, [66] in a study using a multiple baseline design with five participants. Scrupulosity OCD involves having religious or moral obsessions wherein individuals are overly concerned that something they thought or did might be a sin or violation of a religious or moral doctrine. At post-treatment, participants reported a $74 \%$ reduction in self-reported compulsions and a $79 \%$ reduction in avoided valued behaviors. Avoided valued behaviors refer to valued activities that were avoided because of fear that they might trigger the obsessions or compulsions (e.g., praying, going to church or the temple). At 3-month followup, participants reported an $80 \%$ reduction in compulsions and an $87 \%$ reduction in avoided valued behaviors. A preliminary study investigated ACT for youth with OCD using a multiple baseline design [67]. Three adolescents, aged 12 or 13 years, were treated with 8-10 sessions of ACT (without in-session exposure exercises). The primary dependent variable was daily self-monitoring of compulsions. Results indicated a $40 \%$ mean reduction in self-reported compulsions from pre-treatment to post-treatment, with results maintained at 3-month follow-up, thus providing preliminary support for the use of ACT in youth as well.

\section{Mindfulness Based Cognitive Therapy MBCT}

Mindfulness is the awareness that emerges through paying attention on purpose, in the present moment. It is the non judgmental perception of the unfolding of experience moment by moment [68]. Mindfulnessbased Cognitive Therapy (MBCT) is a promising psychosocial group-based relapse prevention program [69]. This programme was originally developed for patients with recurrent depression. However its application to OCD has also been studied. In contrast to traditional cognitive therapy that aims to identify and restructure maladaptive thoughts, MBCT targets the attitude towards thoughts, not their content. That is, in $\mathrm{MBCT}$, patients are taught to carefully observe every arising thought, label it as a thought, try not to judge it and refrain from acting on it compulsively [70]. Preliminary results point towards the efficacy of mindfulness-based treatments for the reduction of OC symptoms. A quasi-experimental study conducted by Hanstede, Gidron, and Nyklícek [71], on a student population found a decline in subclinical OC symptoms after a mindfulness based intervention. A qualitative study of patient experiences undergoing MBCT for OCD has been conducted [70]. The therapeutic intervention program was adapted from a manual on MBCT for depression by Segal, Williams and Teasdale [69]. Cognitive elements of the manual were adapted with the objective of providing a disorder-specific treatment. Mindfulness elements were adopted from the original manual. MBCT was delivered in eight sessions that took place once a week for two hours. Semi-structured interviews were conducted with the 12 participants who had a diagnosis of OCD after the 8 sessions. Researchers found that participants perceived a decline in their obsessive compulsive symptoms, a perceived increase in a feeling of living more actively in the present moment, an increased ability to allow or experience unpleasant emotions, a calmer approach in everyday life and also towards OCD symptoms as well as improved mood and sleep. The results of the study thus provide evidence that patients with OCD find MBCT suitable and helpful. A study was also conducted in India [72] at three points in time: pre-, mid- and post-therapy to evaluate the efficacy of MBCT. Five individuals with a diagnosis of OCD were recruited and treated using MCBT. Results indicated that MBCT was effective in reducing the symptoms in all the patients. This was inspite of the fact that the cases were heterogeneous in nature. Thus, there is preliminary evidence for the applicability of MBCT in the treatment of OCD across multiple cultures.

\section{CONCLUSION}

To summarize, it is seen that metacognition has presence in the theory and treatment of OCD for a number of years but is gaining momentum in recent years in terms of practice and research. Metacognition 
has been identified as being central in the maintenance of OCD in a large number of studies. Many assessment tools known to have good psychometric properties are available to explore the presence and severity of metacognition as part of the therapeutic process and in research. The major aim of therapy for patients with OCD should focus on metacognitive changes. Promising results have been noted from uncontrolled and controlled trials of metacognitive interventions. However, many of the studies had small samples, and shorter follow-ups. Thus, there is a need for more empirical evidence to firmly establish the role of metacognition using large-scale, random-controlled and longer follow-up studies across cultures. The independent contribution of modification of metacognitive beliefs in symptom improvement needs to be further examined. It would be beneficial to examine the specific contribution of various metacognitons to the different OCD subtypes and also to investigate the efficacy of different metacognitive therapies for OCD subtypes. The effectiveness of metacognitive therapies needs to be understood in relation to OCD with different comorbid conditions. Future research could also involve an examination of the applicability of metacognitive therapies to different populations.

\section{REFERENCES}

1. American Psychiatric Association. Diagnostic and Statistical Manual for the Classification of Mental Disorders. 5th ed. Washington DC: Author; 2013.

2. Ruscio AM, Stein DJ, Chiu WT, Kessler RC. The epidemiology of obsessive-compulsive disorder in the National Comorbidity Survey Replication. Mol Psychiatry 2010;15(1):53-63.

3. Flavell JH. Metacognition and cognitive monitoring: A new area of cognitive-developmental inquiry. Am Psychol 1979;34(10):906-11.

4. Wells A. Emotional disorders and metacognition: innovative cognitive therapy. Chichester: Wiley; 2002.

5. Rees CS, Anderson RA. A review of metacognition in psychological models of obsessive-compulsive disorder. Clin Psychol Rev 2013;17(1):1-8.

6. Salkovskis PM. Obsessional-compulsive problems: A cognitive-behavioural analysis. Behav Res Ther 1985;23(5):571-83.

7. Rachman S. Obsessions, responsibility and guilt. Behav Res Ther 1993;31(2):149-54.

8. Rachman S. A cognitive theory of obsessions. Behav ResTher 1997;35(9):793-802.

9. Rachman S. A cognitive theory of obsessions: elaborations. Behav Res Ther 1998;36(4):385-401.

10. Rachman S, Shafran R. Cognitive distortions: thought-action fusion. Clin Psychol Psychother 1999;6(2):80-5.

11. Rachman S, Thordarson DS, Shafran R, Woody SR. Perceived responsibility: Structure and significance. Behav Res Ther 1995;33(7):779-84.

12. Shafran R, Thordarson DS, Rachman S. Thought-action fusion in obsessive compulsive disorder. J Anxiety Disord 1996;10(5):379-91.

13. Shafran R, Rachman S. Thought-action fusion: a review. J Behav Ther Exp Psychiatry 2004;35(2):87-107.

14. Frost RO, Steketee G. Cognitive approaches to obsessions and compulsions: Theory, assessment, and treatment. Elsevier; 2002.

15. OCCWG. Cognitive assessment of obsessive-compulsive disorder. Behav Res Ther 1997;35(7):667-81.

16. OCCWG. Development and initial validation of the obsessive beliefs questionnaire and the interpretation of intrusions inventory. Behav Res Ther 2001;39(8):987-1006.

17. OCCWG. Psychometric validation of the Obsessive Beliefs Questionnaire and the Interpretation of Intrusions Inventory: Part I. Behav Res Ther 2003;41(8):863-78.

18. Clark DA, Purdon C. New Perspectives for a Cognitive Theory of Obsessions. Aust Psychol 1993;28(3):161-7.

19. Wells A, Matthews G. Attention and emotion: a clinical perspective. Hove: Erlbaum; 1994.

20. Wells A. Meta-Cognition and Worry: A Cognitive Model of Generalized Anxiety Disorder. Behav Cog Psychother 1995;23(03):301-20.

21. Wells A, Matthews G. Modelling cognition in emotional disorder: The S-REF model. Behav Res Ther 1996;34(1112):881-8.

22. Gwilliam P, Wells A, Cartwright-Hatton S. Dose meta-cognition or responsibility predict obsessive-compulsive symptoms: a test of the metacognitive model. Clin Psychol Psychother 2004;11(2):137-44.

23. Emmelkamp PMG, Aardema A. Metacognition, specific obsessive-compulsive beliefs and obsessive-compulsive behaviour. Clin Psychol \& Psychother. 1999;6(2):139-45

24. Brown TA, Naragon-Gainey K. Evaluation of the Unique and Specific Contributions of Dimensions of the Triple Vulnerability Model to the Prediction of DSM-IV Anxiety and Mood Disorder Constructs. Behav Ther 2013;44(2):277-92. 
25. Wells A, Papageorgiou C. Relationships between worry, obsessive-compulsive symptoms and meta-cognitive beliefs. Behav Res Ther 1998;36(9):899-913.

26. Janeck AS, Calamari JE, Riemann BC, Heffelfinger SK. Too much thinking about thinking?: metacognitive differences in obsessive-compulsive disorder. J Anxiety Disord 2003;17(2):181-95.

27. Sica C, Steketee G, Ghisi M, Chiri LR, Franceschini S. Metacognitive beliefs and strategies predict worry, obsessivecompulsive symptoms and coping styles: A preliminary prospective study on an Italian non-clinical sample. Clin Psychol Psychother 2007;14(4):258-68.

28. Anand N, Sharma M. Obsessive compulsive beliefs and metacognitions in patients with obsessive compulsive disorder. Indian J Ment Health 2016;3(3):278-86.

29. Solem S, Håland ÅT, Vogel PA, Hansen B, Wells A. Change in metacognitions predicts outcome in obsessivecompulsive disorder patients undergoing treatment with exposure and response prevention. Behav Res Ther 2009;47(4):301-7.

30. Myers SG, Fisher PL, Wells A. An empirical test of the metacognitive model of obsessive-compulsive symptoms: Fusion beliefs, beliefs about rituals, and stop signals. J Anxiety Disord 2009;23(4):436-42

31. Solem S, Myers SG, Fisher PL, Vogel PA, Wells A. An empirical test of the metacognitive model of obsessivecompulsive symptoms: Replication and extension. J Anxiety Disord 2010;24(1):79-86.

32. OCCWG. Psychometric validation of the obsessive belief questionnaire and interpretation of intrusions inventoryPart 2: Factor analyses and testing of a brief version. Behav Res Ther 2005;43(11):1527-42.

33. Tolin DF, Worhunsky P, Maltby N. Are "obsessive" beliefs specific to OCD?: A comparison across anxiety disorders. Behav Res Ther 2006;44(4):469-80.

34. Tolin DF, Woods CM, Abramovitz JS. Relationship between obsessive beliefs and obsessive-compulsive symptoms. Cog Ther Res 2003;27(6):657-69.

35. Cartwright-Hatton S, Wells A. Beliefs about Worry and Intrusions: The Meta-Cognitions Questionnaire and its Correlates. J Anxiety Disord 1997;11(3):279-96.

36. Wells A, Cartwright-Hatton S. A short form of the metacognitions questionnaire: properties of the MCQ-30. Behav Res and Ther 2004;42(4):385-96.

37. Spada MM, Mohiyeddini C, Wells A. Measuring metacognitions associated with emotional distress: Factor structure and predictive validity of the metacognitions questionnaire 30. Person Ind Differ 2008;45(3):238-42.

38. Ramos-Cejudo J, Salguero JM, Cano-Vindel A. Spanish Version of the Meta-Cognitions Questionnaire 30 (MCQ30). The Spanish J Psychol 2013;16 (E95):1-8.

39. Tosun A, Irak M. Adaptation, validity, and reliability of the Metacognition Questionnaire-30 for the Turkish population, and its relationship to anxiety and obsessive compulsive symptoms. Turk J Psychiatry 2008;19:67-80.

40. Quattropani MC, Lenzo V, Mucciardi M, Toffle ME. Psychometric properties of the Italian version of the Short Form of the Metacognitions Questionnaire (MCQ-30). Boll Psicol Appl 2014;269:29-41.

41. Cho Y, Jahng S, Chai S. The Factor Structure and Concurrent Validity of the Korean Version of the Metacognitions Questionnaire 30 (K-MCQ-30). J Clin Psychol 2012;68(3):349-91.

42. Sica C, Coradeschi D, Sanavio E, Dorz S, Manchisi D, Novara C. A study of the psychometric properties of the Obsessive Beliefs Inventory and Interpretations of Intrusions Inventory on clinical Italian individuals. J Anxiety Disord 2004;18(3):291-307.

43. Clark D, Purdon C, Wang A. The Meta-Cognitive Beliefs Questionnaire: development of a measure of obsessional beliefs. Behav Res Ther 2003;41(6):655-69.

44. Wells A, Gwilliam, PDH, \& Cartwright-Hatton S. The thought fusion instrument. Unpublished scale, University of Manchester.2001

45. Fisher PL, Wells A. Metacognitive therapy for obsessive-compulsive disorder: A case series. J Behav Ther Exp Psychiatry 2008;39(2):117-32.

46. Rassin E, Merckelbach H, Muris P, Schmidt H. The thought-action fusion scale: further evidence for its reliability and validity. Behav Res Ther 2001;39(5):537-44.

47. Yorulmaz OCA, Yılmaz A, Gençöz T. Psychometric properties of the Thought-Action Fusion Scale in a Turkish sample. Behav Res Ther 2004;42(10):1203-14.

48. Smári J, Hólmsteinsson HE. Intrusive Thoughts, Responsibility Attitudes, Thought-Action Fusion, And Chronic Thought Suppression In Relation To Obsessive-Compulsive Symptoms. Behav Cog Psychother 2001;29(1):13-20.

49. Fisher PL. Obsessive Compulsive Disorder: A Comparison of CBT and the Metacognitive Approach. Int J Cog Ther 2009;2(2):107-22.

50. Wells A, Simons M. Metacognitive therapy. John Wiley \& Sons, Ltd.; 2009.

51. Rees CS, Koesveld KEV. An open trial of group metacognitive therapy for obsessive-compulsive disorder. J Behav Ther Exp Psychiatry 2008;39(4):451-58.

52. Andouz Z, Dolatshahi B, Moshtagh N, Dadkhah A. The efficacy of metacognitive therapy on patients suffering from pure obsession. Iranian J Psychiatry 2012;7(1):11-21. 
53. Simons M, Schneider S, Herpertz-Dahlmann B. Metacognitive Therapy versus Exposure and Response Prevention for Pediatric Obsessive-Compulsive Disorder. Psychother and Psychosom 2006;75(4):257-64.

54. Shareh H, Gharraee B, Atef-Vahid MK, Eftekhar M. Metacognitive therapy (MCT), fluvoxamine, and combined treatment in improving obsessive-compulsive, depressive and anxiety symptoms in patients with obsessivecompulsive disorder (OCD). Iranian J Psychiatry Behav Sci 2010;4(2):17-25.

55. Moritz S, Jelinek L, Hauschildt M, Naber D. How to treat the untreated: effectiveness of a self-help metacognitive training program (myMCT) for obsessive-compulsive disorder. Dialogues Clin Neurosci 2010;12(2):209-20.

56. O'connor K, Koszegi N, Aardema F, Niekerk JV, Taillon A. An Inference-Based Approach to Treating ObsessiveCompulsive Disorders. Cog Behav Prac 2009;16(4):420-9.

57. O'Connor K, Pélissier Marie Claude., Aardema F. Beyond Reasonable Doubt: Reasoning Processes in Obsessive Compulsive Disorder and Related Disorders. Chirchester: John Wiley \& Sons; 2005.

58. O'connor KP, Aardema F, Bouthillier D, Fournier S, Guay S, Robillard S, et al. Evaluation of an Inference- Based Approach to Treating Obsessive- Compulsive Disorder. Cog Behav Ther 2005;34(3):148-63.

59. Aardema F, O'connor K. Dissolving the tenacity of obsessional doubt: Implications for treatment outcome. J Behav Ther Exp Psychiatry 2012;43(2):855-61.

60. Visser HA, Megen HV, Oppen PV, Eikelenboom M, Hoogendorn AW, Kaarsemaker M, et al. Inference-Based Approach versus Cognitive Behavioral Therapy in the Treatment of Obsessive-Compulsive Disorder with Poor Insight: A 24-Session Randomized Controlled Trial. Psychother Psychosom 2015;84(5):284-93.

61. Hayes SC, Barnes-Holmes D, Roche B. Relational frame theory: A post-Skinnerian account of human language and cognition. Springer Science \& Business Media.; 2001.

62. Hayes SC, Strosahl K, Wilson KG. Acceptance and commitment therapy: an experiential approach to behavior change. New York: Guilford Press; 1999.

63. Twohig MP. The Application of Acceptance and Commitment Therapy to Obsessive-Compulsive Disorder. Cog Behav Prac 2009;16(1):18-28.

64. Twohig MP, Hayes SC, Masuda A. Increasing Willingness to Experience Obsessions: Acceptance and Commitment Therapy as a Treatment for Obsessive-Compulsive Disorder. Behav Ther 2006;37(1):3-13.

65. Twohig MP, Hayes SC, Plumb JC, Pruitt LD, Collins AB, Hazlett-Stevens H. A randomized clinical trial of acceptance and commitment therapy versus progressive relaxation training for obsessive-compulsive disorder. J Consult Clin Psychol 2010;78(5):705-16.

66. Dehlin JP, Morrison KL, Twohig MP. Acceptance and Commitment Therapy as a Treatment for Scrupulosity in Obsessive Compulsive Disorder. Behav Modif 201337(3):409-30.

67. Armstrong AB, Morrison KL, Twohig MP. A Preliminary Investigation of Acceptance and Commitment Therapy for Adolescent Obsessive-Compulsive Disorder. J Cog Psychother 2013;27(2):175-90.

68. Kabat-Zinn J. Mindfulness-Based Interventions in Context: Past, Present, and Future. Clin Psychol Sci Prac 2003;10(2):144-56.

69. Segal ZV, Teasdale JD, Williams JMG. Mindfulness-based cognitive therapy for depression: a new approach to preventing relapse. New York: Guilford Press; 2002.

70. Hertenstein E, Rose N, Voderholzer U, Heidenreich T, Nissen C, Thiel N, et al. Mindfulness-based cognitive therapy in obsessive-compulsive disorder - A qualitative study on patients' experiences. BMC Psychiatry 2012;12(1):185.

71. Hanstede M, Gidron Y, Nyklíček I. The Effects of a Mindfulness Intervention on Obsessive-Compulsive Symptoms in a Non-Clinical Student Population. J Nerv Ment Dis 2008;196(10):776-89.

72. Sharma M, Goyal A, Salam AKP, Kumar D. Mindfulness-Based Cognitive Behaviour Therapy in OCD: A Case Series. Arch Indian Psychiatry 2012;14(2):9-15.

$* * * * * * * * * * * * * * * * * * * * * *$

Acknowledgements - Nil

Source of Funding - Nil

Conflict of Interest - Nil 